\title{
Lack of Association of the NPAS2 Gene Ala394Thr Polymorphism (rs2305160:G>A) with Risk of Chronic Lymphocytic Leukemia
}

\author{
Sobia Rana ${ }^{1,2}$, Adeela Shahid ${ }^{1,3}$, Hafeez Ullah $^{4}$, Saqib Mahmood ${ }^{5 *}$
}

\begin{abstract}
Background: NPAS2 is a product of the circadian clock gene. It acts as a putative tumor suppressor by playing an important role in DNA damage responses, cell cycle control and apoptosis. Chronic lymphocytic leukemia (CLL) appears to be an apoptosis related disorder and alteration in the NPAS2 gene might therefore be directly involved in the etiology of CLL. Here, the Ala394Thr polymorphism (rs2305160:G $>$ A) in the NPAS2 gene was genotyped and melatonin concentrations were measured in a total of seventy-four individuals, including thirty-seven CLL cases and an equal number of age- and sex-matched healthy controls in order to examine the effect of NPAS2 polymorphism and melatonin concentrations on CLL risk in a Pakistani population. Materials and Methods: Genotyping of rs2305160:G>A polymorphism at NPAS2 locus was carried out by amplification refractory mutation system-polymerase chain reaction (ARMS-PCR). Melatonin concentrations were determined by enzyme linked immunosorbent assay (ELISA). Statistical analysis was performed using Statistical Package for Social Sciences software. Results: Our results demonstrated no association of the variant Thr genotypes (Ala/ Thr and Thr/Thr) with risk of CLL. Similarly, no association of rs2305160 with CLL was observed in either females or males after stratification of study population on a gender basis. Moreover, when the subjects with CLL were further stratified into shift-workers and non-shift-workers, no association of rs2305160 with CLL was seen in either case. However, significantly low serum melatonin levels were observed in CLL patients as compared to healthy subjects $(\mathbf{p}<\mathbf{0 . 0 5})$. Also, lower melatonin levels were seen in shift-workers as compared to non-shift-workers $(p<0.05)$. There was no significant difference $(p>0.05)$ in the melatonin levels across NPAS2 genotypes in all subjects, subjects with CLL who were either shift workers or non-shift-workers. General Linear Model (GLM) univariate analysis revealed no significant association $(\mathrm{p}>\mathbf{0 . 0 5})$ of the rs2305160 polymorphism of the NPAS2 gene with melatonin levels in any of the groups. Conclusions: While low melatonin levels and shift-work can be considered as one of the risk factors for CLL, the NPAS2 rs2305160 polymorphism does not appear to have any association with risk of CLL in our Pakistani population.
\end{abstract}

Keywords: NPAS2 - rs2305160 - polymorphism - genotypes - cancer - CLL

Asian Pac J Cancer Prev, 15 (17), 7169-7174

\section{Introduction}

NPAS2 (Neuronal PAS domain protein 2) is the largest $(176.68 \mathrm{~kb})$ of nine human core circadian genes that regulate multiple biological processes by running 24-h circadian rhythm. It maps on chromosome 2 at $2 \mathrm{q} 11.2$ and encodes for a member of the basic helix-loop-helixPAS class of transcription factors (Zhou et al., 1997). It is a functional analogue of CLOCK, and is expressed in the mammalian forebrain and several peripheral tissues. Like CLOCK, it forms heterodimer with BMAL1 and binds to E-box sequences in the promoter regions of target circadian genes, thereby activating their expression and regulating circadian rhythm (DeBruyne et al., 2007; Bertolucci et al., 2008). Previous reports indicated the involvement of NPAS2 in tumorigenesis, by regulating PER2 that can act as tumour suppressor ( Fu and Lee, 2003), and by suppressing transcription of c-Myc that is an oncogene (Fu et al., 2002). Now recent studies describe NPAS2 as a putative tumor suppressor playing an important role in DNA damage response, cell cycle control and apoptosis by activating different downstream genes (Chen-Goodspeed and Lee, 2007; Hoffman et al., 2008; Yi et al., 2009). Similarly, emerging data have revealed the role of NPAS2 as a risk biomarker in human cancers as significant associations were found between a missense polymorphism (SNP rs2305160:G>A, Ala394Thr) in NPAS2 and risk of breast cancer (Zhu et al., 2008), prostate cancer (Chu et al., 2007) and non-Hodgkin's lymphoma (Zhu et al., 2007). A more recent study suggests that the

${ }^{1}$ Department of Physiology and Cell Biology, ${ }^{4}$ Department of Allied Health Sciences, ${ }^{5}$ Department of Human Genetics \& Molecular Biology, University of Health Sciences, Lahore, ${ }^{3}$ Department of Physiology, Shalamar Medical and Dental College Lahore, Punjab, ${ }^{2}$ Panjwani Center for Molecular Medicine and Drug Research (PCMD), International Center for Chemical and Biological Sciences (ICCBS), University of Karachi, Sindh, Pakistan*For correspondence: sqb_medgen@yahoo.com,medgen@uhs.edu.pk 
genotype of NPAS2 (Ala/Ala, Ala/Thr, and Thr/Thr) may serve as a marginal prognostic biomarker in addition to its association with breast cancer risk (Yi C et al., 2010). Furthermore, most recently, SNP rs2305160 in the NPAS2 gene showed significant associations with overall death risk in hepatocellular carcinoma (HCC) patients with a significant trend of increasing death risk with increasing number of unfavorable genotypes (Yuan et al., 2014).

The public NCBI dbSNP database (www.ncbi.nlm.nih.gov/SNP) holds 560 single nucleotide polymorphisms (SNPs) identified in the NPAS2 gene, of which the SNP rs2305160 $(\mathrm{G}>\mathrm{A})$ is the only non-synonymous mutation altering an amino acid at codon 394, Ala394Thr.

Chronic lymphocytic leukemia (CLL) is marked by proliferation and accumulation of morphologically mature but immunologically dysfunctional lymphocytes in blood, bone marrow and lymphoid tissues. In East Europe and USA the disease incidence is high while in Asia and Africa CLL is rare. The age-adjusted incidence rate of CLL in the UK and USA is 4.2 per 100,000 per year (Howlader et al., 2012). Studies on the racial and geographic distribution show that CLL is 20-30 times commoner in Europe, Australasia and North American white and black populations than in India, China and Japan (Sgambati et al., 2001).

No particular genetic change has been associated with this disease so far. Specifically, CLL is not associated with reciprocal balanced chromosomal translocations, but rather with specific deletions (Do “ hner et al., 1999). This information points towards the loss of presently unidentified tumor suppressor genes. CLL cells show low proliferative rate and a prolonged life span indicating that their principal change may be a defect in apoptosis (Caligaris-Cappio and Hamblin, 1999). Since CLL seems to be an apoptosis related disorder and circadian genes regulate many biological pathways including cell proliferation and apoptosis, their alteration may be directly involved in the etiology of CLL.

The current case-control study involves genotyping of rs2305160 polymorphism of NPAS2 gene in a total population of 74 individuals including 37 CLL patients and equal number of age- and sex-matched healthy controls in order to find any association between this nonsynonymous polymorphism and risk of CLL. Moreover, serum melatonin levels of CLL patients and healthy controls were determined to find any association between melatonin (considered as a circadian biomarker) levels and rs2305160 polymorphism of NPAS2 gene.

\section{Materials and Methods}

The study was carried out at University of Health Sciences Lahore, Pakistan, after approval of the University's Ethical Committee and Review Board. The study involved a total population of 74 individuals including 37 diagnosed cases of CLL and equal number of age- and sex-matched healthy individuals as controls. The informed consents were obtained from both study and control groups. The patients were recruited from oncology departments of various local tertiary care hospitals of Lahore. Clinical diagnosis was based on standard morphological and immunophenotypic criteria.

Blood samples were drawn from CLL patients and healthy controls in EDTA-coated purple top (for subsequent DNA isolation) and red top (for subsequent serum isolation) vacutainer tubes (BD, USA). Melatonin concentrations were determined by using human melatonin ELISA kit (Cusabio Biotech, China). Genomic DNA was extracted from whole blood using genomic DNA purification kit (Fermentas, USA). Genotyping of rs2305160:G $>$ A polymorphism at NPAS2 locus was carried out by amplification refractory mutation system polymerase-chain reaction (ARMSPCR). For this purpose, wild-allele-specific forward primer A (5'-ACCTCGGCAGCACTTTATCG-3'), mutant-allele-specific forward primer B (5'-ACCTCGGCAGCACTTTATCA-3') and a common reverse primer (5' - TGCTTGGCAATGAATGGATA-3') were used. The thermocyclic conditions involved initial denaturation at $95^{\circ} \mathrm{C}$ for $5 \mathrm{~min}$ followed by 35 cycles, each of denaturation at $94^{\circ} \mathrm{C}$ for $30 \mathrm{sec}$, annealing at $61.4^{\circ} \mathrm{C}$ for $30 \mathrm{sec}$, extension at $72^{\circ} \mathrm{C}$ for $30 \mathrm{sec}$, with a final extension at $72^{\circ} \mathrm{C}$ for $10 \mathrm{~min}$. The PCR products were subjected to agarose (2\%) gel electrophoresis and results were recorded under gel documentation system. A PCR product of $340 \mathrm{bp}$ obtained from Primer pair A only corresponds to homozygous wild-type genotype (GG or Ala/Ala) whereas PCR product obtained from primer-pair B only corresponds to homozygous mutant genotype (AA or Thr/Thr). A PCR product, obtained from both A and B primer pairs, specifies heterozyguos genotype GA or Ala/Thr (Figure 1).

Statistical analysis was performed using Statistical Package for Social Sciences software (SPSS Inc. Chicago, IL, USA, version 17.0). Genotype and allele frequencies at SNP locus were checked for compliance with HardyWeinberg equilibrium $(p>0.05)$. Allele frequencies were calculated by gene counting. Chi-square $\left(\chi^{2}\right)$ test was used to determine the significant differences of genotype and allelic frequencies between CLL patients and control groups. Association of rs2305160 variant with CLL was determined by Pearson Chi-square test using codominant, dominant and recessive model followed by Yates correction. Odds ratio (ORs) with $95 \%$ confidence

M

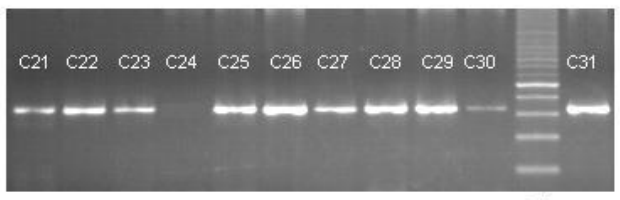

A

M

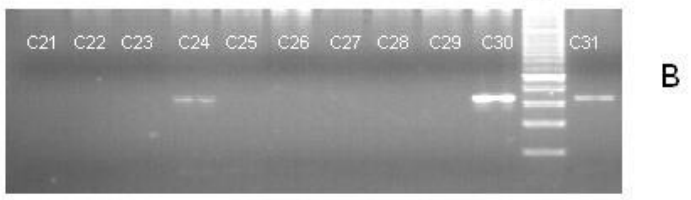

Figure 1. ARMS-PCR Product of CLL Patients (C21-C31) Amplified from Primer Pair A and B. M: 100 bp Marker, A: Primer pair A, B: Primer pair B, Wild type homozygous (GG): C21, C22, C23, C25, C26, C27, C28, C29, Mutant homozygous (AA): C24, Heterozygous (GA): C30, C31 
intervals $(\mathrm{CI})$ was calculated to determine the relative risk of CLL associated with different genotypes. Student's t-test was applied to make comparisons of melatonin levels between CLL patients and healthy controls and also between shift-workers and non-shift-workers. Difference between groups was determined by applying One Way Anova followed by Post Hoc Test. General Linear Model (GLM) was applied to determine the significant association of rs2305160 polymorphism of NPAS2 gene with melatonin levels. A p-value of $<0.05$ was considered statistically significant.

\section{Results}

A total of 74 subjects between 45-85 years of age were involved in the study. Among a total population of 74 subjects, $50 \%$ were CLL patients and other $50 \%$ were age- and sex-matched healthy controls. In each group, $73 \%$ were males and $27 \%$ were females. Furthermore, among CLL patients, $30 \%$ were shift-workers whereas all healthy controls were non-shift-workers. The characteristics of study population are given in Table 1 .

All CLL and control subjects were genotyped for rs2305160 variant of NAPS2 gene. The genotypes of NPAS2 rs2305160 variant were in Hardy-Weinberg equilibrium $(\mathrm{p}=0.14)$ in the control group. Among CLL

patients, $12.5 \%$ were homozygous AA, $24.3 \%$ were heterozygous GA and $59.5 \%$ were homozygous GG. In the control group, the frequency of AA genotype was 19\%, GA $35.1 \%$ and GG $45.9 \%$ (Table 2). We did not find any significant difference in distribution of genotypes and allele frequencies for rs2305160 variant between CLL patients and their age and sex-matched healthy controls ( $P>0.05)$. Also, no association of rs2305160 variant with increase or decrease risk of CLL was found using codominant $(\mathrm{p}=0.45)$, dominant $(\mathrm{OR}=0.82,95 \% \mathrm{CI}=0.24-$ $2.75, \mathrm{p}=0.50)$ and recessive $(\mathrm{OR}=0.57,95 \% \mathrm{CI}=0.23$ $1.45, \mathrm{p}=0.17)$ model. Furthermore, no association was observed between allelic variants ( $\mathrm{G}$ or $\mathrm{A})$ and risk of CLL (OR=0.68, 95\%CI=0.34-1.37, p=0.19) (Table 2).

Among CLL patients, nearly $30 \%$ of the subjects were shift-workers. When data was stratified in shift-

Table 1. Characteristics of Study Population

\begin{tabular}{lcc}
\hline & CLL Patients $(\mathrm{n}=37)$ & Control Subjects $(\mathrm{n}=37)$ \\
\hline Age (mean \pm SD) & $62.81 \pm 10.84$ & $62.81 \pm 10.84$ \\
Males & $27(73 \%)$ & $27(73 \%)$ \\
Females & $10(27 \%)$ & $10(27 \%)$ \\
Shift-workers & $11(30 \%)$ & - \\
Non-shift-workers & $26(70 \%)$ & - \\
\hline *Data are presented as number of cases and percentages are given in parenthesis \\
and age as mean \pm SD
\end{tabular}

Table 2. Genotype and Allele Distribution of the NPAS2 Polymorphism in CLL Patients and Control Subjects

\begin{tabular}{|c|c|c|c|c|c|c|c|}
\hline \multirow[t]{2}{*}{ Genotype } & & \multicolumn{3}{|c|}{ All Subjects $(n=74)$} & \multicolumn{3}{|c|}{ CLL Patients $(n=37)$} \\
\hline & & $\begin{array}{l}\text { CLL } \\
(\mathrm{n}=37)\end{array}$ & $\begin{array}{l}\text { Controls } \\
(n=37)\end{array}$ & $\chi^{2} / \mathrm{P} / \mathrm{OR}(\mathrm{CI})$ & $\begin{array}{c}\text { Shift-Workers } \\
\quad(\mathrm{n}=11)\end{array}$ & $\begin{array}{c}\text { Non-Shift- } \\
\text { Workers }(n=26)\end{array}$ & $\chi^{2} / \mathrm{P} / \mathrm{OR}(\mathrm{CI})$ \\
\hline \multicolumn{8}{|c|}{ Co-dominant } \\
\hline \multirow[t]{3}{*}{ Model } & GG & $22(59.5 \%)$ & $17(45.9 \%)$ & $1.45 / 0.45$ & $7(63.6 \%)$ & $15(57.7 \%)$ & $0.59 / 0.74$ \\
\hline & GA & $9(24.3 \%)$ & $13(35.1 \%)$ & & $3(27.3 \%)$ & $6(23.1 \%)$ & \\
\hline & AA & $6(16.2 \%)$ & $7(19 \%)$ & & $1(9.1 \%)$ & $5(19.2 \%)$ & \\
\hline \multicolumn{8}{|c|}{ Dominant } \\
\hline \multirow[t]{2}{*}{ Model } & $\mathrm{GA} / \mathrm{AA}$ & $15(40.5 \%)$ & $20(54.1 \%)$ & $1.36 / 0.17 /$ & $4(36.4 \%)$ & $11(42.3 \%)$ & $0.11 / 0.7 /$ \\
\hline & GG & $22(59.5 \%)$ & $17(45.9 \%)$ & $0.57(0.23-1.45)$ & $7(63.6 \%)$ & $15(57.7 \%)$ & $0.77(0.18-3.35)$ \\
\hline \multicolumn{8}{|c|}{ Recessive } \\
\hline \multirow[t]{2}{*}{ Model } & AA & $6(16.2 \%)$ & $7(18.9 \%)$ & $0.09 / 0.50 /$ & $1 \quad(9.1 \%)$ & $5(19.2 \%)$ & $0.08 / 0.41 /$ \\
\hline & GG/GA & $31(83.8 \%)$ & $30(81.1 \%)$ & $0.82(0.24-2.75)$ & $10(90.9 \%)$ & $21(80.8 \%)$ & $0.42(0.04-4.08)$ \\
\hline \multirow[t]{2}{*}{ Alleles } & $\mathrm{G}$ & $53(71.6 \%)$ & $47(63.5 \%)$ & $1.11 / 0.19 /$ & $17(77.3 \%)$ & $36(69.2)$ & $0.49 / 0.34 /$ \\
\hline & A & $21(28.4 \%)$ & $27(36.5 \%)$ & $0.68(0.34-1.37)$ & $5(22.7 \%)$ & $16(30.8 \%)$ & $0.66(0.20-2.10)$ \\
\hline
\end{tabular}

Table 3. Genotype and Allele Distribution of the NPAS2 Polymorphism in Male and Female Subjects of CLL Patients and Control Subjects

\begin{tabular}{|c|c|c|c|c|c|c|c|}
\hline \multirow[t]{2}{*}{ Genotype } & & \multicolumn{3}{|c|}{ Male Subjects $n=54$} & \multicolumn{3}{|c|}{ Female Subjects $n=20$} \\
\hline & & $\begin{array}{c}\text { CLL } \\
(\mathrm{n}=27)\end{array}$ & $\begin{array}{c}\text { Controls } \\
(n=27)\end{array}$ & $\overline{\chi^{2} / \mathrm{P} / \mathrm{OR}(\mathrm{CI})}$ & $\begin{array}{c}\text { CLL } \\
(\mathrm{n}=10)\end{array}$ & $\begin{array}{l}\text { Controls } \\
(n=10)\end{array}$ & $\chi^{2} / \mathrm{P} / \mathrm{OR}(\mathrm{CI})$ \\
\hline \multicolumn{8}{|c|}{ Co-dominant } \\
\hline \multirow[t]{3}{*}{ Model } & GG & $15(55.6 \%)$ & $11(40.74 \%)$ & $1.20 / 0.54$ & $7(70 \%)$ & $6(60 \%)$ & $1.41 / 0.49$ \\
\hline & GA & $8(29.6)$ & $11(40.74 \%)$ & & $1(10 \%)$ & $3(30 \%)$ & \\
\hline & AA & $4(14.8 \%)$ & $5(18.52 \%)$ & & $2(20 \%)$ & $1(10 \%)$ & \\
\hline \multicolumn{8}{|l|}{ Dominant } \\
\hline \multirow[t]{2}{*}{ Model } & GA/AA & $12(44.4 \%)$ & $16(59.3 \%)$ & $1.19 / 0.20 /$ & $3(30 \%)$ & $4(40 \%)$ & $0.22 / 0.6 /$ \\
\hline & GG & $15(55.6 \%)$ & $11(40.7 \%)$ & $0.55(0.18-1.61)$ & $7(70 \%)$ & $6(60 \%)$ & $0.64(0.10-4.09)$ \\
\hline \multicolumn{8}{|l|}{ Recessive } \\
\hline \multirow[t]{2}{*}{ Model } & $\mathrm{AA}$ & $4(14.8 \%)$ & $5(18.5 \%)$ & $0.13 / 0.7 /$ & $2(20 \%)$ & $1(10 \%)$ & $0.39 / 0.53 /$ \\
\hline & GG/GA & $23(85.2 \%)$ & $22(81.5 \%)$ & $0.76(0.18-3.22)$ & $8(80 \%)$ & $9(90 \%)$ & $2.25(0.17-29.7)$ \\
\hline \multirow[t]{2}{*}{ Alleles } & $\mathrm{G}$ & $38(70.4 \%)$ & $33(61.1 \%)$ & $1.03 / 0.31 /$ & $15(75 \%)$ & $15(75 \%)$ & $0.13 / 0.64 /$ \\
\hline & A & $16(29.6 \%)$ & $21(38.9 \%)$ & $1.51(0.67-3.33)$ & $5(25 \%)$ & $5(25 \%)$ & $1(0.23-4.18)$ \\
\hline
\end{tabular}


Table 4. Comparisons of Melatonin Levels of CLL and Control Subjects, Shift-Workers and Non-ShiftWorkers and Across Genotypes of NPAS2

\begin{tabular}{|c|c|c|c|c|}
\hline & \multicolumn{3}{|c|}{ Melatonin Levels of CLL Patient and Controls } & \multirow{2}{*}{ p value } \\
\hline & $\begin{array}{c}\text { CLL Patients }(n=37) \\
\text { mean } \pm \text { SEM }\end{array}$ & \multicolumn{2}{|c|}{$\begin{array}{c}\text { Normal/Controls }(\mathrm{n}=37) \\
\text { mean } \pm \text { SEM }\end{array}$} & \\
\hline Melatonin(ng/dL) & $101 \pm 3.65$ & \multicolumn{2}{|c|}{$141 \pm 7.77$} & $<0.0001^{*}$ \\
\hline \multicolumn{5}{|c|}{ Melatonin Levels among CLL Patients } \\
\hline Melatonin(ng/dL) & \multicolumn{3}{|c|}{$\begin{array}{cc}\text { Shift Workers }(n=11) & \text { Non Shift Workers }(n=26 \\
\text { mean } \pm \text { SEM } & \text { mean } \pm \text { SEM } \\
\text { g/dL) } 86.6 \pm 5.6 & 107 \pm 4.1\end{array}$} & $\begin{array}{l}\text { 6) } \\
\mathrm{p} \text { value } \\
<0.0001^{*}\end{array}$ \\
\hline \multirow{2}{*}{ Melatonin(ng/dL) } & \multicolumn{3}{|c|}{ Melatonin Levels Across NPAS2 Genotypes } & \multirow[b]{2}{*}{$\mathrm{p}$ value } \\
\hline & $\begin{array}{c}\mathrm{GG} \\
\text { mean } \pm \text { SEM }\end{array}$ & $\begin{array}{c}\text { GA } \\
\text { mean } \pm \text { SEM }\end{array}$ & $\begin{array}{c}\mathrm{AA} \\
\text { mean } \pm \text { SEM }\end{array}$ & \\
\hline All Subjects & $116 \pm 5.61$ & $129 \pm 9.91$ & $120 \pm 14.6$ & 0.51 \\
\hline CLL & $101 \pm 5.49$ & $98.1 \pm 6.28$ & $107 \pm 4.8$ & 0.74 \\
\hline Shift Workers & $81.1 \pm 8.10$ & $99.7 \pm 1.0$ & $94.12 \pm 3.64$ & 0.42 \\
\hline Non Shift Workers & s $110 \pm 5.77$ & $97.7 \pm 8.21$ & $113 \pm 3.44$ & 0.33 \\
\hline
\end{tabular}

*Melatonin levels are presented as mean \pm SEM and were compared by t-test and One Way ANOVA. $\mathrm{P}<0.05$ was considered statistically significant

workers and non-shift-workers within the CLL group, no significant difference in genotype and allele frequencies was observed. Moreover, no association of rs2305160 polymorphism was found with increase or decrease risk of CLL in shift-workers and non-shift-workers within the CLL group (Table 2). When the data was further stratified according to gender, no significant difference in genotype and allele frequencies of rs2305160 gene variant in both male and female groups was observed. Similarly, no association of rs2305160 variant of NPAS2 with CLL was observed in either males or females (Table 3 ).

Melatonin levels of CLL patients were significantly low $(\mathrm{p}<0.0001)$ as compared to control subjects (Table 4). When the subjects with CLL were further stratified into shift-workers and non-shift-workers, melatonin levels of shift workers were significantly lower $(\mathrm{p}<0.0001)$ as compared to those of non-shift workers (Table 4). There was no significant difference $(\mathrm{p}>0.05)$ in the melatonin levels across NPAS2 genotypes in all subjects, subjects with CLL, CLL subjects who were shift workers and those who were non-shift-workers (Table 4). GLM Univariate analysis revealed no significant association $(\mathrm{p}>0.05)$ of rs2305160 polymorphism of NPAS2 gene with melatonin levels in any of the groups (Table 4).

\section{Discussion}

Deregulation of the circadian clock may disturb the expression of clock-controlled genes and have a profound influence on organ function (Luo et al., 2012). NPAS2 may not only directly regulate other genes in the circadian regulatory system (Asher and Schibler, 2006; DeBruyne et al., 2007) but genes involved in cancerrelated biological pathways are also influenced by it (Hoffman et al., 2008; Yi et al., 2009). This could explain the observed functional effect of NPAS2 knockdown on cell cycle progression and DNA repair capacity, as well as the epidemiologic evidence indicating an association between polymorphisms in NPAS2 and cancer risk (Zhu et al., 2007; 2008). The current study was intended to find any association between non-synonymous polymorphism of NPAS2 gene, rs2305160:G $>\mathrm{A}$, and risk of CLL.

CLL is a disease of middle-aged to older people, with a median age of 65 to 70 years at presentation (Oscier et al., 2004). The incidence increases with age and is higher in men than women and higher in Caucasians than in other racial groups. Men are approximately twice as likely to be diagnosed with CLL as women (Howlader et al., 2012). Similar statistics in terms of age and male dominance among CLL patients were found in Asian countries like China and Iran (Kermani et al., 2007; Mozaheb et al., 2012). These statistics are almost in line with those observed in the current study representing CLL patients mostly from elderly population with a mean age of $62.81 \pm 10.84($ mean \pm SD) years. Similarly, male dominance among CLL population was observed with male to female ratio of 2.7:1. Moreover, among CLL patients, $\sim 30 \%$ were found shift-workers. Shift-work has been reported as a risk factor for cancer in many studies (Straif et al., 2007).

NPAS2 rs2305160 polymorphism has also been studied in some other cancers including Non-Hodgkin's lymphoma (NHL), breast and prostate cancer. Zhu et al. (2007) in a genetic association study examined Ala394Thr polymorphism in NPAS2 gene and its impact on NHL risk. After stratification by subtypes of B-cell lymphoma, the genotypic frequencies observed in B-cell chronic lymphocytic leukemia/prolymphocytic leukemia/small lymphocytic lymphoma (CLL/PLL/SLL) (44.4\% Ala/ Ala, $46.3 \% \mathrm{Ala} / \mathrm{Thr}, 9.3 \% \mathrm{Thr} / \mathrm{Thr}$ ) are different from those found in CLL group of the current study $(59.5 \%$ Ala/Ala, 24.3\% Ala/Thr, $16.2 \% \mathrm{Thr} / \mathrm{Thr}$ ). Also, genotypic frequencies in control group of current study (45.9\% Ala/ Ala, 35.1\% Ala/Thr, 19\% Thr/Thr) are different from genotypic frequencies in control group of the previous study (41.4\% Ala/Ala, $46.1 \% \mathrm{Ala} / \mathrm{Thr}, 12.5 \% \mathrm{Thr} / \mathrm{Thr})$. Zhu et al. observed significantly decreased risks between NPAS2 variant Thr genotypes (Ala/Thr and Thr/Thr) and B-cell chronic lymphocytic leukemia/prolymphocytic leukemia/small lymphocytic lymphoma (CLL/PLL/SLL) after stratification by subtypes of B-cell lymphoma (Zhu et al., 2007). Another study found that women with the heterozygous Ala394Thr genotype were significantly associated with breast cancer risk compared to those with the common homozygous Ala394Ala (Zhu et al., 2008). However, using the study population from China, Wang and colleagues did not observe the association between rs2305160 and breast cancer development (variant $\mathrm{T}$ allele frequency: 0.230 in the cases and 0.236 in the controls, $\mathrm{P}=0.683$; adjusted $\mathrm{OR}=0.99,95 \% \mathrm{CI}=0.83-1.18$ in the dominant genetic model) (Wang et al., 2011). Furthermore, in a population-based case-control study conducted in China, among men with less insulin resistance (IR), a reduced prostate cancer risk was associated with the NPAS2-variant A allele that was not seen among men with greater IR (Chu et al., 2007). The current study is in line with study by Wang et al. (2011) and contrary to studies by Zhu et al. $(2007,2008)$ as current study showed no significant association of rs2305160 Even no significant association between rs2305160 with risk of CLL was observed after gender stratification of whole population 
and stratification of CLL group in shift-workers and nonshift-workers. This difference in results of current study with those of studies by Zhu et al. $(2007,2008)$ may be attributable to the difference in type of study populations (racial difference).

Melatonin is known to protect against oxidative injury of toxins and radiations with its free radical scavenging capacity (Kucuktulu, 2012). The current study showed low melatonin levels in CLL patients compared to their healthy controls. This observation is in agreement with other studies that also provide evidence for low levels of melatonin as risk factors in other cancers (Bartsch et al., 1992; Schernhammer and Hankinson, 2009). Current study also reports low melatonin levels in shift-workers as compared to non-shift-workers within the CLL group Mirick and Davis (2008) proposed that circulating melatonin levels can be considered as a biomarker of circadian disruption and has been associated with nightshift-work and exposure to light-at-night. Exposure to light-at-night, including disturbance of the circadian rhythm, possibly mediated via the melatonin synthesis and clock genes, has been suggested as a contributing cause of many cancers (Costa et al., 2010). However, Japan Collaborative Cohort Study for evaluation of cancer showed no association between shift-work and any site of cancer (Fujino, 2007).

Based on above results and discussion, it can be speculated from the current study that low melatonin levels and shift-work can be considered as risk factors for cancer like CLL. However, low melatonin levels do not appear to have any relation with NPAS2 rs2305160 polymorphism. Similarly, this polymorphism may not be associated with increasing or decreasing risk of CLL in Pakistani population. The above speculation is based on results obtained from a relatively small sample size, as CLL is rare in this region of the world. However, attempts can be made to replicate this study in a larger sample size to further validate this speculation.

\section{Acknowledgements}

We thank Higher Education Commission of Pakistan for providing funding for this research work.

\section{References}

Asher G, Schibler U (2006). A CLOCK-less clock. Trends Cell Biol, 16, 547-49.

Bartsch C, Bartsch H, Schmidt A, et al (1992). Melatonin and 6-sulfatoxymelatonin circadian rhythms in serum and urine of primary prostate cancer patients: evidence for reduced pineal activity and relevance of urinary determinations. Clin Chim Acta, 209, 153-67.

Bertolucci C, Cavallari N, Colognesi I, et al (2008). Evidence for an overlapping role of CLOCK and NPAS2 transcription factors in liver circadian oscillators. Mol Cell Biol, 28, 3070-75.

Chen-Goodspeed M, Lee CC (2007). Tumor suppression and circadian function. J Biol Rhythms, 22, 291-98.

Chu LW, Zhu Y, Yu K, et al (2008). Variants in circadian genes and prostate cancer risk: a population-based study in China. Prostate Cancer Prostatic Dis, 11, 342-48.
Costa G, Haus E, Stevens R (2010). Shift work and cancer considerations on rationale, mechanisms, and epidemiology. Scand J Work Environ Health, 36, 163-79.

DeBruyne JP, Weaver DR, Reppert SM (2007). CLOCK and NPAS2 have overlapping roles in the suprachiasmatic circadian clock. Nat Neurosci, 10, 543-45.

Do “ hner H, Stilgenbauer K, Dohner M (1999). Chromosome aberrations in B-cell chronic lymphocytic leukemia: reassessment based on molecular cytogenetic analysis. $J$ Mol Med, 77, 266-81.

Fujino Y (2007). Occupational factors and mortality in the Japan Collaborative Cohort Study for Evaluation of Cancer (JACC). Asian Pac J Cancer Prev, 8, 97-104.

Fu L, Lee CC (2003). The circadian clock: pacemaker and tumour suppressor. Nat Rev Cancer, 3, 350-61.

Fu L, Pelicano H, Liu J, Huang P, Lee C (2002). The circadian gene Period2 plays an important role in tumor suppression and DNA damage response in vivo. Cell, 111, 41-50.

Hoffman AE, Zheng T, Ba Y, Zhu Y (2008). The circadian gene NPAS2, a putative tumor suppressor, is involved in DNA damage response. Mol Cancer Res, 6, 1461-68.

Howlader N, Noone AM, Krapcho M, et al (2012). SEER Cancer Statistics Review 1975-2009 (Vintage 2009 Populations). National Cancer Institute, Bethesda, MD. http:// seer. cancer.gov.

Kermani IA, Dehdilani M, Dolatkhah R (2007). Chronic lymphocytic leukemia in the recent 10 years and treatment effects of Fludarabin. Asian Pac J Cancer Prev, 8, 367-71.

Kucuktulu E (2012). Protective effect of melatonin against radiation induced nephrotoxicity in rats. Asian Pac J Cancer Prev, 13, 4101-05.

Luo Y, Wang F, Chen LA, et al (2012). Deregulated expression of cry1 and cry2 in human gliomas. Asian Pac J Cancer Prev, 13, 5725-28.

Mirick DK, Davis S (2008). Melatonin as a biomarker of circadian dysregulation. Cancer Epidemiol Biomarkers Prev, 17, 3306-13.

Mozaheb Z, Hasanzadeh NazarAbadi MH, Aghaee MA (2012). Chronic lymphocytic leukemia and prognostic factors. Asian Pac J Cancer Prev, 13, 3009-13.

Oscier D, Fegan C, Hillmen P, et al (2004). British Committee for Standards in Haematology. Guidelines on the diagnosis and management of chronic lymphocytic leukaemia. $\mathrm{Br} \mathrm{J}$ Haematol, 125, 294-317.

Schernhammer ES, Hankinson SE (2009). Urinary melatonin levels and postmenopausal breast cancer risk in the Nurses' Health Study cohort. Cancer Epidemiol Biomarkers Prev, 18, 74-9.

Sgambati MT, Linet MS, Devesa SS (2001). Chronic Lymphocytic Leukemia: Epidemiological, Familial, and Genetic Aspests. In 'Chronic Lymphoid Leukemias', Eds Cheson BD, Marcel Dekker, New York pp 33-62.

Straif K, Baan R, Grosse Y (2007). Carcinogenicity of shiftwork, painting, and fire-fighting. Lancet Oncol, 8, 1065-66.

Wang F, Hu Z, Yang R, et al (2011). A variant affecting miRNAs binding in the circadian gene neuronal PAS domain protein 2 (NPAS2) is not associated with breast cancer risk. Breast Cancer Res Treat, 127, 769-75.

Yi CH, Zheng T, Leaderer D, Hoffman A, Zhu Y (2009). Cancerrelated transcriptional targets of the circadian gene NPAS2 identified by genome-wide ChIP-on-chip analysis. Cancer Lett, 284, 149-56.

Yi C, Mu L, de la Longrais IA, et al (2010). The circadian gene NPAS2 is a novel prognostic biomarker for breast cancer. Breast Cancer Res Treat, 120, 663-69.

Yuan P, Wang S, Zhou F, et al (2014). Functional polymorphisms in the NPAS2 gene are associated with overall survival 


\section{Sobia Rana et al}

in transcatheter arterial chemoembolization-treated hepatocellular carcinoma patients. Cancer Sci, 105, 825-32.

Zhou YD, Barnard M, Tian H, et al (1997). Molecular characterization of two mammalian bHLH-PAS domain proteins selectively expressed in the central nervous system. Proc Natl Acad Sci USA, 94,713-18.

Zhu Y,LeadererD, Guss C, et al (2007). Ala394Thr polymorphism in the clock gene NPAS2: a circadian modifier for the risk of non-Hodgkin's lymphoma. Int J Cancer, 120, 432-35.

Zhu Y, Stevens RG, Leaderer D, et al (2008). Non-synonymous polymorphisms in the circadian gene NPAS2 and breast cancer risk. Breast Cancer Res Treat, 107, 421-5. 